\title{
How to determine critical Marangoni number in half floating zone convection
}

\author{
Y. L. YAO, F. LIU and W. R. HU \\ Institute of Mechanics, Chinese Academy of Sciences, Beijing 100080, China
}

(Received for publication 17 July 1995)

\begin{abstract}
In the present paper, the coordinated measurements of the temperature profile inside the liquid bridge and the boundary variation of free surface, in addition to other quantities, were obtained in the same time for the half floating zone convection. The results show that the onset of free surface oscillation is earlier than the one of temperature oscillation during the increasing of applied temperature difference, and the critical Marangoni numbers, defined usually by temperature measurement, are larger than the one defined by free surface measurement, and the difference depends on the volume of liquid bridge. These results induce the question, "How to determine experimentally the critical Marangoni number?" Copyright
\end{abstract} (C) 1996 Elsevier Science Ltd.

\section{INTRODUCTION}

Thermocapillary convection, especially the onset of oscillation, is a typical subject in material processing [1-5], and has important meanings in many theories such as microgravity fluid physics, heat and mass transfer, non-linear science, hydrodynamics and thermodynamics [6-10]. The floating zone convection has been studied extensively, and is a typical process of thermocapillary convections. Half floating zone convection has been adopted as a model for deep research on the mechanism of the thermocapillary convection and the research is also related to the floating zone crystal growth. A critical applied temperature difference $(\Delta T)_{\mathrm{c}}$ or the critical Marangoni number $(M a)_{\mathrm{c}}$ has been introduced to define the onset of oscillation, and the Marangoni number is defined as follows

$$
M a=-\frac{(\mathrm{d} \sigma / \mathrm{d} T) \delta T l}{\kappa \mu},
$$

where $\sigma, \kappa$ and $\mu$ are, respectively, the surface tension, thermal diffusion coefficiency and viscosity, $T$ is temperature and $l$ is the height of liquid bridge.

The critical state of thermocapillary convection describes the onset of oscillation, and is usually defined by the variation of temperature profile inside the liquid bridge, or the variation of flow pattern. The critical applied temperature difference $(\Delta T)_{\mathrm{c}}$ is experimentally determined by the onset moment of oscillation, for example, the temperature measured by inserted thermocouple shows the transition of temperature profiles from steady distribution into oscillatory distribution during the increasing of applied temperature difference $\Delta T$ between the upper rod and lower rod, and the critical value $(\Delta T)_{c}$ associates with the value of $\Delta T$ at the onset moment of oscillation. The flow pattern, measured usually by tracer particle method, shows that the onset of oscillation associates with the transition of flow pattern from symmetric and steady convection into asymmetric and oscillatory convection. However, the resolution of tracer particle measurement is not sensitive enough to judge if the onset of flow pattern oscillation is either earlier or later than the onset of temperature oscillation.

Recently, some non-contact methods of optical diagnostics were developed to measure the transition and oscillation feature [11-14]. A light sheet passed through the transparent liquid bridge, and gave clearly an interference line pattern on the screen. The onset of free surface oscillation may associate with the transition of a steady pattern of interference lines to an oscillatory pattern of interference lines [11]. A microvideo system was designed to measure the radii variations in one cross-section of the liquid bridge [12, 13], and the time resolution of measurement can be high enough in comparison with the typical oscillatory period of $1 \mathrm{~s}$ in order of magnitude for a small liquid bridge with a diameter of a few millimeters. Furthermore, the surface wave on an area of free surface was also measured by a griding mask method $[14,15]$, that is, a laser beam passed through a griding mask and then projected on a area of free surface, and the configuration of free surface and then, the free surface wave in the area may be demonstrated by the distorted grid image which reflected from the free surface and projected on the screen. Coordinated measurement of more physical quantities including free surface deformation and oscillation, flow pattern and temperature have been completed $[14,15]$, these results are favourable for studying the detailed process of onset of oscillation.

In the present paper, the onset of oscillation depending on the volume of liquid bridge and the heating rate was systematically studied by coordinated 


\section{NOMENCLATURE}

$\begin{array}{ll}A & \text { geometrical aspect ratio } \\ d_{\mathrm{o}} & \text { diameter of rod } \\ d_{\mathrm{m}} & \text { minimum diameter of liquid bridge } \\ k & \text { element number of image in horizontal } \\ & \text { direction } \\ l & \text { height of liquid bridge } \\ M a \quad \text { Marangoni number } & \\ (M a)_{\mathrm{c}}^{\mathrm{A}} \quad \text { critical Marangoni number due to } & \text { free surface oscillation } \\ (M a)_{\mathrm{c}}^{\mathrm{T}} \quad \text { critical Marangoni number due to } & \text { temperature oscillation } \\ n \quad \text { number of measurement points }\end{array}$

$r \quad$ radial coordinate in cylindrical coordinate system

$r_{0} \quad$ radial coordinate of free surface

$T$ temperature.

Greek symbols

$\begin{array}{ll}\kappa & \text { thermal diffusion coefficiency } \\ \mu & \text { dynamical viscosity } \\ \sigma & \text { surface tension } \\ \Delta r & \text { variation of free surface } \\ \Delta T & \text { applied temperature difference. }\end{array}$

measurement. The results show that, the onset of free surface oscillation is earlier than the onset of temperature oscillation. These results imply that, there will be different critical applied temperature difference or different critical Marangoni number given by a different physical quantity.

The method of coordinated measurement will be discussed in the next section, and, then, the experimental results in Section 3. The last section is discussion and conclusion.

\section{COORDINATED MEASUREMENT}

The experimental system of coordinated measurement including free surface boundary, free surface wave, flow pattern and temperatures inside the liquid bridge was developed for drop shaft experiment on oscillatory convection in a half floating zone, where the facility can be operated by the telescience method $[16,17]$. In the present paper, a part function of the system was used for studying the onset process, as shown schematically in Fig. 1, and the free surface boundary variation, measured by a microscopic video system and temperatures in the liquid bridge measured by inserted thermocouples will be emphasized to show the different onset moment of oscillation in details in the present paper.

A liquid bridge of 10 CST silicon oil was used as the experimental medium. Co-axial upper and lower rods had the same diameter of $d_{o}=4 \mathrm{~mm}$, and the geometrical aspect was $A=l / d_{\mathrm{o}}=0.8$. The cases of upper rod being heated will be studied, and the heating rate may be controlled as low as $0.5^{\circ} \mathrm{C} \mathrm{min}-1$. The very low heating rate is beneficial for studying the different onset processes of oscillations related to different physical quantities. The temporal resolution of measurement should be better than the typical time of oscillatory period, which is $1 \mathrm{~s}$ in order of magnitude for a small liquid bridge of a few millimeters in diameter.

The free surface deformation and vibration are important quantities to measure the features of oscillatory convection [7]. A sensitive system of microscopic measurement was especially developed. The amplification ratio of microscopic a CCD camera is 10 times in a view field of $0.795 \mathrm{~mm}$ (height) $\times 0.645$

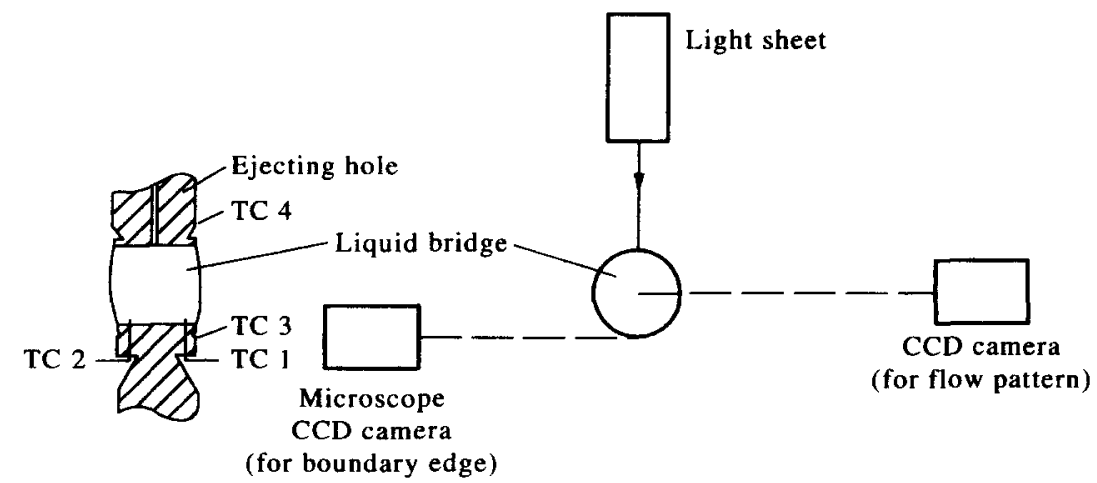

Fig. 1. Schematic diagram of coordinated measurement including temperature measurement by thermocouples (left), free surface boundary measurement by microscope CCD camera and flow pattern (right). where TC means thermocouple. 
$\mathrm{mm}$ (width), and the resolution of the CCD element is $512 \times 512$ lines. So, the measurement error of the $\mathrm{CCD}$ camera is

$$
(\delta s)_{\mathrm{CCD}} \approx 0.645 \mathrm{~mm} /(512 \times 2)=0.63 \mu \mathrm{m}
$$

in the horizontal direction. The relative positions of 80 points in the view field were measured at the same moment during the data analyses, and the real measured boundary of free surface was given after the adjustment by spline curves. Therefore, the real measurement error is

$$
\delta s=(\delta s)_{\mathrm{CCD}}(k / n)=0.07 \mu \mathrm{m},
$$

where $n$ is the number of measurement points and $k$ is element number covered by the image in the horizontal direction. The amplitude of free surface vibration is a few micrometers, which is larger than the measured error of at least one order of magnitude. The CCD camera has a speed of $0.04 \mathrm{~s}$ per picture, and the temporal resolution will be less than $0.04 \mathrm{~s}$, which is much smaller than the oscillatory period.

The thermocouples of $0.03 \mathrm{~mm}$ in diameter were used to measure the temperature distribution inside the liquid bridge, and two thermocouples were inserted from the lower rod at radius $r=1.35 \mathrm{~mm}$ with $180^{\circ}$ azimuthal angle difference into the liquid bridge at 0.3 and $0.5 \mathrm{~mm}$ above the lower rod, respectively. The measurement error of thermocouple itself may be usually less than $0.1^{\circ} \mathrm{C}$ for a temperature range smaller than $100^{\circ} \mathrm{C}$. The thermocouple signal of electrical voltage will be amplified and then transferred by 12 bit-A/D plane into a digital program of computer. The errors introduced by amplifier and A/D transfer are smaller than the error of the thermocouple, and the system error of temperature measurement is $0.1^{\circ} \mathrm{C}$ in order of magnitude. However, the time resolution of thermocouple measurement is typically $0.01 \mathrm{~s}$ in order of magnitude, and the delay due to the electrical and computer processes has the same order of magnitude, which is also much smaller than the typical oscillatory period of 1 $s$ in order of magnitude. Therefore, the thermocouple measurement is suitable to analyze the onset of oscillation during the increase of applied temperature difference.

The experiments were completed by the tele-operation technique. The volume of the liquid bridge and the heating rate could be designed in a program for a fixed rod diameter $d_{\mathrm{o}}$ and geometrical aspect $A$. The upper rod was heated gradually at a heating rate to increase the applied temperature difference. A different heating rate, including a very low rate of $0.5^{\circ} \mathrm{C}$ $\min ^{-1}$, was designed in the experiments. The lower heating rate will be useful in judging if the onset of oscillation for one critical quantity is earlier or later than the other one.

\section{EXPERIMENTAL RESULTS}

The onset and offset of oscillations were obtained for different heating rates and different volumes of the

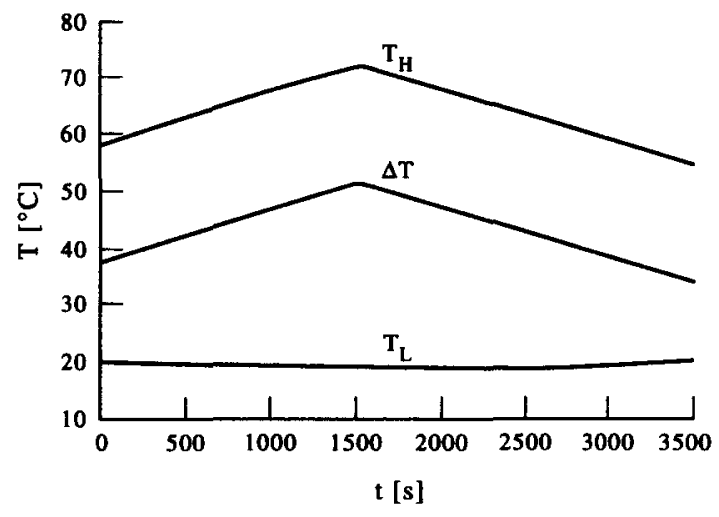

Fig. 2. Variation history of applied temperature difference with a very slow heating rate of $0.5^{\circ} \mathrm{C} \mathrm{min}-1$ and $d_{\mathrm{m}} / d_{\mathrm{o}}=0.8$ for studying the onset and offset oscillation.

liquid bridge. As an example of experimental cases, the temporal evolution of upper rod temperature, lower rod temperature and applied temperature difference was shown in Fig. 2 for a very slow heating rate and $d_{\mathrm{m}} / d_{\mathrm{o}}=0.8$, where $d_{\mathrm{m}}$ was the minimum diameter of the liquid bridge and $d_{\mathrm{m}} / d_{\mathrm{o}}$ was used to measure the relative volume of the liquid bridge. The onset oscillation and offset oscillation were given, respectively, in Figs 3 and 4 for a very slow heating rate of $0.5^{\circ} \mathrm{C} \mathrm{min}{ }^{-1}$ and the variation of the free surface boundary was given by the relative radius $A$

$$
\Delta r=r-r_{\mathrm{o}}
$$

where $r$ and $r_{\mathrm{o}}$ were, respectively, the real radius of the free surface and a constant value near the steady state. The results show clearly that, the onset of free surface oscillation is earlier than the one of temperature oscillation, and the offset of oscillation for a free surface is later than the one for temperature. It means that the free surface variation is a critical parameter which is more sensitive in comparison with the temperature variation in the oscillatory process. The onset of oscillation for a normal heating rate such as $0.14^{\circ} \mathrm{s}^{-1}$ was also obtained, as shown typically in Fig. 5 for $d_{\mathrm{m}} / d_{\mathrm{o}}=0.7$, and similar conclusions of the early onset of oscillation and late offset of oscillation for the free surface boundary in comparison with temperature were also obtained.

The onset of oscillation given by the free surface boundary and temperature have generally different moments of several minutes for the cases of low heating rate and a few minutes for the cases of normal heating rate. Henceforth, the critical applied temperature difference defined by the temperature oscillation will be several degrees larger than the one defined by the free surface oscillation, as shown in Fig. 6 for cases of normal heating rate. Similar distributions were obtained also for the cases of slow heating rate. It could be seen that the critical Marangoni number $(M a)_{\mathrm{c}}^{\mathrm{T}}$ or critical applied temperature difference $(\Delta T)_{c}^{\mathbf{T}}$ defined usually by the temperature variation of the liquid bridge is larger than the one 


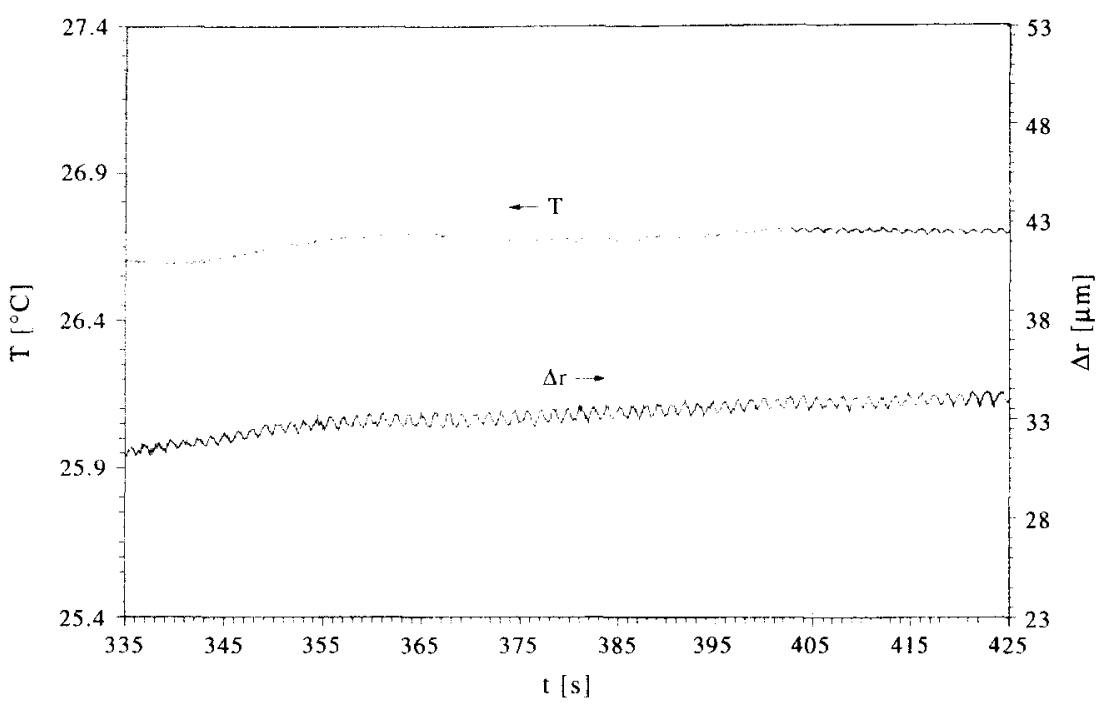

Fig. 3. The onset processes of oscillations for temperature (upper curve and left coordinate) and free surface radii (lower curve and right coordinate) in the case of heating rate given by Fig. 2 .

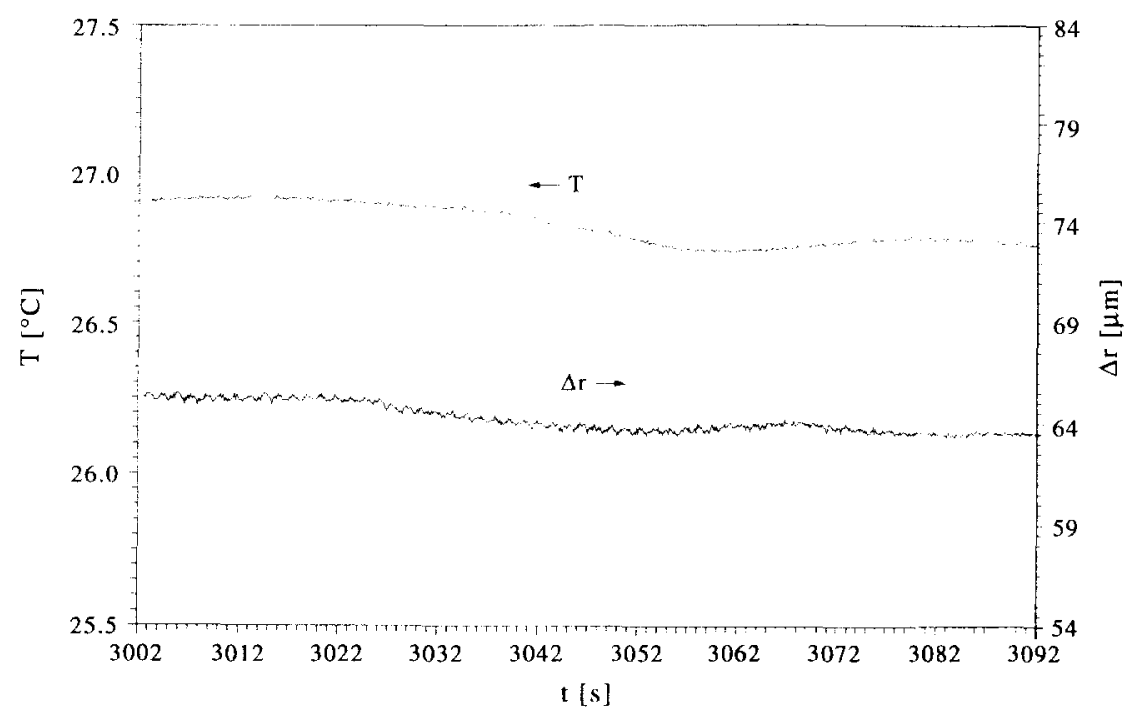

Fig. 4. The offset processes of oscillation for temperature and free surface radii in cases of heating rate given by Fig. 2 .

$(M a)_{\mathrm{c}}^{\mathrm{A}}$ or $(\Delta T)_{\mathrm{c}}^{\mathrm{A}}$ defined by free surface variation, for example,

$$
(M a)_{i}^{\top}=(1+0.07)(M a)_{c}^{\mathrm{A}}
$$

for the case $d_{\mathrm{m} /} / d_{\mathrm{o}}=0.8$.

It should be pointed out that the temperature measurements at different positions of the liquid bridge may also give different critical Marangoni numbers $(M a)_{\mathrm{C}}^{\mathrm{T}}$. However, the delays of the onset of temperature oscillation due to the different positions are small, usually only a few s for a very slow heating rate, and the difference of critical Marangoni number is small enough to be neglected.

\section{DISCUSSIONS AND CONCLUSIONS}

The transition from steady flow into oscillation for thermocapillary convection is a complete process. From the viewpoint of theory, the mechanism of the onset of oscillation or the mechanism of instability is still an open problem. From the viewpoint of experiment, there are several physical quantities which 


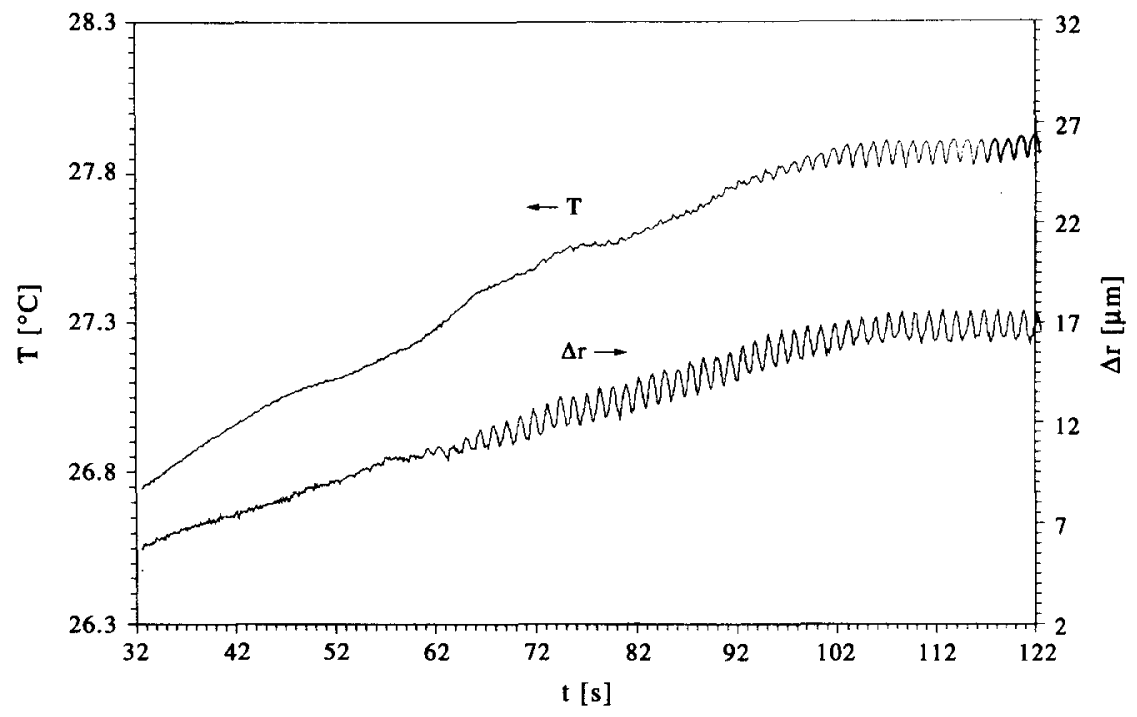

Fig. 5. The onset processes of oscillation for temperature and free surface radii with normal heating rate of $0.14^{\circ} \mathrm{C} \mathrm{s}^{-1}$.

could be used to describe the transition process, and in most cases, the temperature has been selected. According to the results of the present paper, it seems that the temperature is not sensitive enough in comparison with the boundary of the free surface. Therefore, the coordinated measurement to involve more physical quantities and to measure accurately will be important for discovery of the intrinsic mechanism on the onset of oscillation.

Obviously the time delay between the free surface boundary and temperature for the onset and offset of oscillation depends on the heating rate, and the longer the delay, the lower the heating rate. Therefore, a very low heating rate will easily observe the different onset

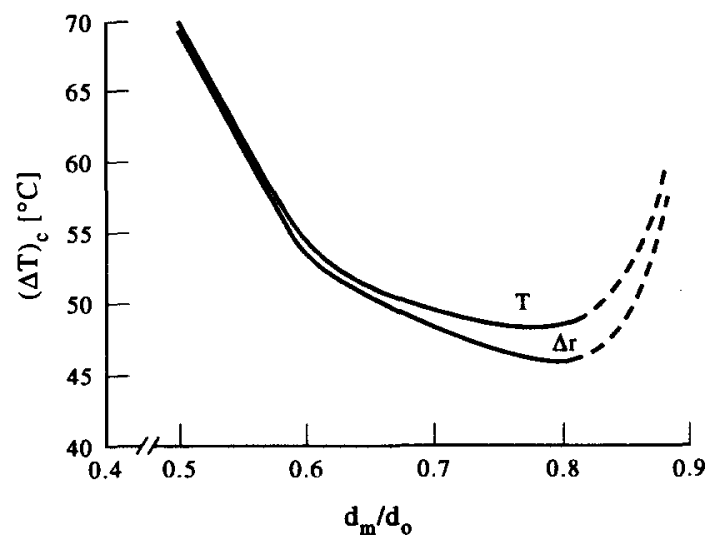

Fig. 6. The distributions of critical applied temperature $(\Delta T)_{\mathrm{c}}^{\mathrm{T}}$ (upper curve) and $(\Delta T)_{\mathrm{c}}^{\mathrm{A}}$ (lower curve) depending on the radii ratio $d_{\mathrm{m}} / d_{0}$ of slender liquid bridge in cases of normal heating rate $0.14^{\circ} \mathrm{C} \mathrm{s}^{-1}$. moment of oscillation for different quantities. However, the differences of critical applied temperature defined by temperature and free surface boundary for different heating rates have similar values of several degrees, however, most experiments concentrating in the temperature measurement have a heating rate which is not low enough. The critical state determined by temperature measurement is relatively later, this conclusion implies that temperature oscillation of the liquid bridge may be induced by other physical quantities, for example the free boundary oscillation.

Based on the experimental results, the oscillatory feature of physical quantities have different amplitudes in order of magnitude. The flow pattern transfer obviously from steady and symmetric flow into oscillatory and asymmetric convection, the oscillatory component of velocity value has the same order of magnitude as the steady velocity, and the perturbation theory cannot be applied to analyze the velocity field during the onset of oscillation. However, the temperature oscillation and free boundary satisfy the perturbation condition, and the ratios of their oscillatory components to their mean components are $10^{-1}, 10^{-2}$ and $10^{-3}$ in order of magnitude, respectively. So, the transition process is a strong nonlinear problem, although the temperature and free boundary are small in perturbation. It seems that after the free surface is excited to oscillation first, there needs to be a relatively longer time delay for exciting the temperature oscillation in the liquid bridge because of the small amplitude of free surface oscillation in order of magnitude. The connection between the free surface oscillation and the temperature oscillation is still not quite clear, and needs to be studied in the future.

The results of the present paper shows that, the free 
surface oscillation is an important critical parameter, which is more sensitive in comparison with the temperature of the liquid bridge. It seems that the coordinated measurement to involve more physical quantities will be important in discovering the mechanism of oscillation. The free surface deformation and oscillation as one of the sensitive critical quantities has been studied in the present paper. The configuration of the free surface relates closely with the pressure distribution near the free surface, and the pressure distribution, which is related to the velocity profile, should be oscillated together with the free surface oscillation. It seems that the pressure distribution should be measured in the next step.

The critical Marangoni number $(M a)_{c}^{\mathrm{T}}$ has been discussed over the last 20 years, and was determined by the temperature measurement in most cases. Many theoretical models were based on the assumption that the free surface was not deformed and oscillated. The conclusion of the present paper emphasized the importance of free surface deformation and oscillation, which defined a Marangoni number $(M a)_{c}^{\mathrm{A}}$ as smaller than the one given by temperature. This result implied that more studies should be completed experimentally and theoretically before we can understand the mechanism of the transition process.

\section{REFERENCES}

1. C. E. Chang and W. R. Wilcox, J. Crystal Growth $\mathbf{2 8 , 8}$ (1975).

2. D. Schwabe and A. Scharmann, J. Crystal Growth 46, 124 (1979).

3. C.-H. Chun, J. Crystal Growth 48, 600 (1980).

4. Y. Kamotani, S. Ostrach and M. Vargas, J. Crystal Growth 66, 83 (1984).

5. W. R. Hu, J. Z. Shu, R. Zhou and Z. M. Yang, J. Crystal Growth 42, 379 (1994).

6. S. H. Davis, Ann. Rev. Fluid Mech. 19, 403 (1987).

7. S. Ostrach, C. Kamotoni and L. Lai, Phys. Chem. Hydrodyn. 6, 599 (1985)

8. W. R. Hu, Acta Astronaut 18, 593 (1990).

9. H. C. Kuhlmann and H. J. Rath, J. Fluid Mech. 247, 247 (1993).

10. W. R. Hu and Z. M. Tang, Int. J. Heat Mass Transfer 37, 1563 (1994).

11. Z. H. Cao, J. C. Xie, Z. M. Tang and W. R. Hu, Adv. Space Res. 11, 163 (1991).

12. Z. H. Cao, H. T. You, Z. M. Tang and W. R. Hu, $A d v$. Space Res. 11, 229 (1991).

13. W. R. Hu, H. T. You and Z. H. Cao, Sci. China A 35, 1101 (1992).

14. J. Z. Shu, Y. L. Yao and W. R. Hu. Sci. China A, 36, 326 (1993).

15. J. Z. Shu, Y. L. Yao and W. R. Hu, Micrograv. Sci. Technol. 7, 83 (1994).

16. Y. L. Yao, J. Z. Shu, J. C. Xie and W. R. Hu, Adv. Space Res. 16, 215 (1995).

17. Y. L. Yao, J. Z. Shu, J. C. Xie, W. R. Hu, A. Hirata, S. 1. Nishizawa and M. Sakurai, AIAA 95-0816 (1995) 